\title{
Bis-4-halophenyl-pyrimidines and -1,2,4,5-tetrazines
}

\author{
Alan R. Katritzky ${ }^{* a}$, Jadwiga Soloducho ${ }^{b}$, and Sergei Belyakov ${ }^{\mathrm{c}}$ \\ ${ }^{a}$ Center for Heterocyclic Compounds, Department of Chemistry, University of Florida, \\ Gainesville, Florida 32611, USA \\ ${ }^{b}$ Wroclaw, University of Technology, I-4, Wybrzeze Wyspianskiego 27, Poland ${ }^{c}$ Guilford \\ Pharmaceuticals Inc., 6611 Tributary Street, Baltimore, Maryland 21224 \\ E-mail: katritzky@chem.ufl.edu
}

(received 24 Aug 99; accepted 13 Feb 00; published on the web 21 Feb 00)

\section{Introduction}

For an ongoing project, we required six-membered heterocycles di-substituted with $p$-halophenyl groups. The preparation of such pyrimidines and tetrazines is described in this note.

\section{Results and Discussion}

Synthesis of 4,6-bis-(4-halophenyl)-2-undecylpyrimidines 7a,b. Shyrina et al. ${ }^{1}$ described the preparation of pyrimidines in yields of $20-70 \%$ by the oxidative coupling of chalcones with acetamidine or benzamidine in DMSO or its mixtures with toluene or xylene in the presence of molecular sieves at elevated temperatures. Based on these results, we commenced our preparation of pyrimidines $\mathbf{7 a , b}$ from chalcones $\mathbf{3 a , b}$. The dichloro- chalcone $\mathbf{3 b}$ is commercially available from Lancaster Synthesis, Inc. The diiodochalcone 3a was prepared by first reducing the acid chloride 1 with $\mathrm{NaBH}_{4}$ in pyridine, using the general method developed by Babler, ${ }^{2}$ to give 4-iodobenzaldehyde 2 in 53\% yield (Scheme 1).<smiles>O=C(Cl)c1ccc(I)cc1</smiles>

1

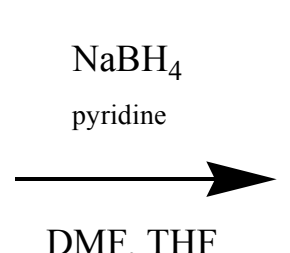

DMF, THF<smiles>O=Cc1ccc(I)cc1</smiles>

2

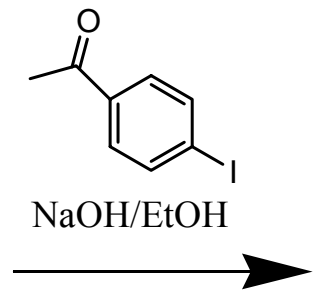

3a

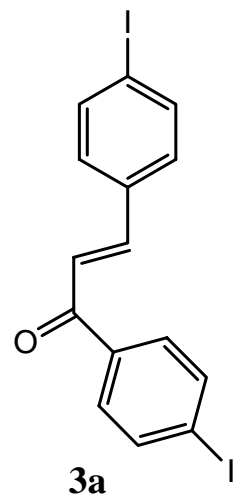

Scheme 1 
Condensation of $\mathbf{2}$ with 4-iodoacetophenone in the presence of base gave the chalcone 3a in $53 \%$ yield. The preparation of dodecylamidine hydrochloride 6 (Scheme 2) was achieved by adapting a procedure ${ }^{3}$ previously reported for several lower aliphatic amidines. Thus, nitrile 4 was treated with hydrogen chloride in $\mathrm{EtOH}$ to afford the imino ester 5 (yield 76\%) which was, without further purification, converted into amidine 6 by treatment with ammonia in EtOH at $70{ }^{\circ} \mathrm{C}$. The amidine 6 was isolated as its hydrochloride salt in $79 \%$ yield. Condensations of chalcones 3a,b with amidine hydrochloride 6 in hot $\left(80{ }^{\circ} \mathrm{C}\right)$ DMSO were followed by column chromatography purification which gave the corresponding 4,6-bis-(4-iodophenyl)-2undecylpyrimidine (7a) and 4,6-bis-(4-chlorophenyl)-2-undecylpyrimidine (7b) in 72\% and 63\% yields, respectively.

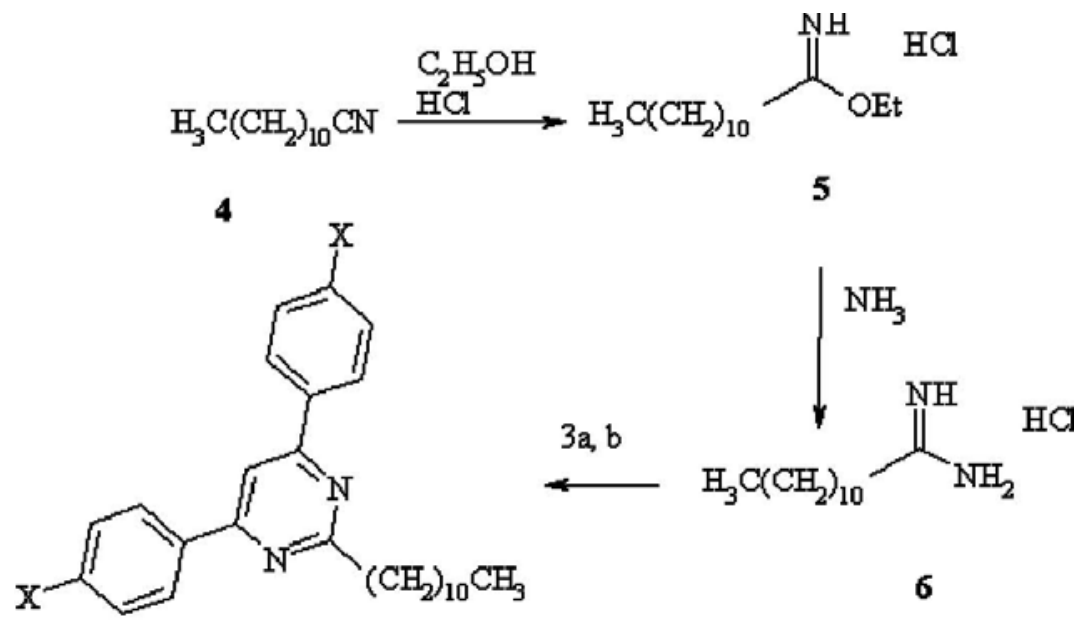

$7 \mathbf{a}, \mathbf{b}$

$$
\begin{aligned}
& 7 \mathrm{a}(\mathrm{X}=\mathrm{D}) \\
& 7 \mathrm{~b}(\mathrm{X}=\mathrm{C} 1)
\end{aligned}
$$

\section{Scheme 2}

Synthesis of 3,6-Bis-(4-halophenyl)-1,2,4,5-tetrazines. The general method of Pinner ${ }^{4}$ was modified to develop a practical preparation of the tetrazines 12 and 13. 4-Aminobenzonitrile (9) prepared in $60 \%$ yield from 4-nitrobenzonitrile (8) as previously reported ${ }^{5}$ (Scheme 3), was cyclized by heating with anhydrous hydrazine (98\%) into the previously unreported 3,6-bis-(4aminophenyl)-1,2-dihydro-1,2,4,5-tetrazine (10) in 51\% yield. Compound $\mathbf{1 0}$ was oxidized to the corresponding tetrazine 11 by treatment with hydrogen peroxide (4\%) following a literature procedure. $^{5}$ 3,6-Bis-(4-aminophenyl)-1,2,4,5-tetrazine (11) was isolated by column chromatography in $18 \%$ yield. Under the conditions shown in the Scheme 4, diamine 11 was converted by a Sandmeyer reaction, in a procedure similarly to that used for the iodination of 2bromo-3,6-dimethoxyaniline ${ }^{6}$ into 3,6- bis-4-iodophenyl-1,2,4,5-tetrazine (12). 


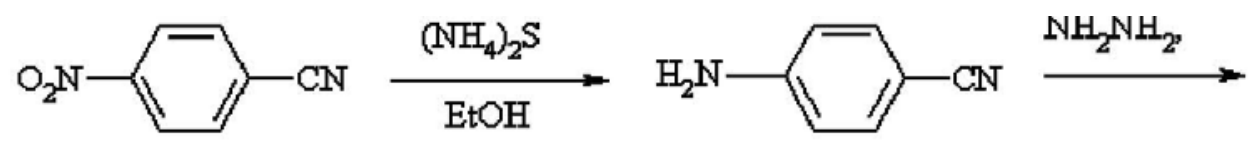

8

9

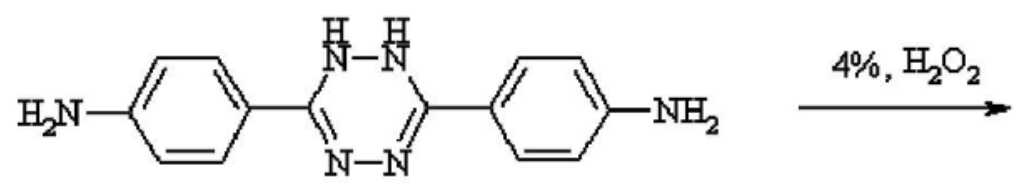

10
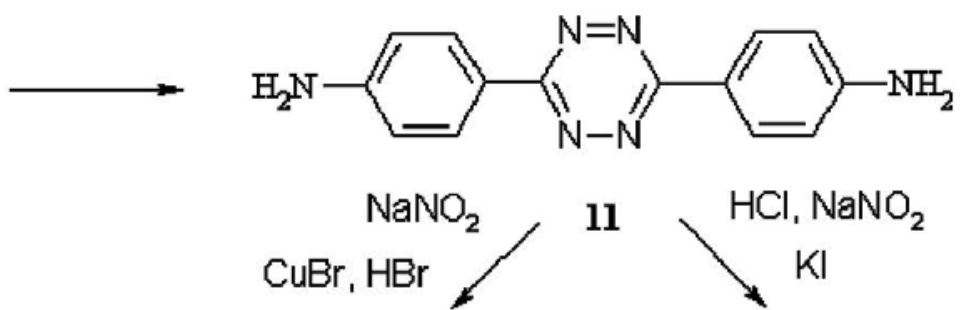

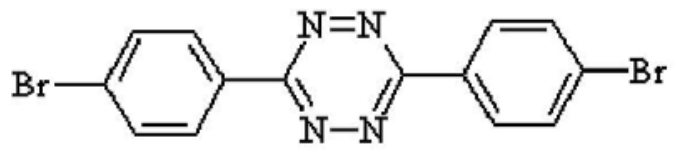

13

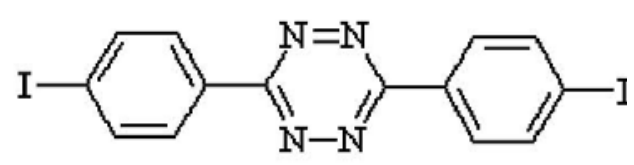

12

\section{Scheme 3}

An analogous Sandmeyer reaction was used to synthesize 3,6-bis-(4-bromophenyl)-1,2,4,5tetrazine (13). 3,6-Bis-(4-aminophenyl)-1,2,4,5-tetrazine 11 was reacted with $\mathrm{NaNO}_{2} / \mathrm{HBr} / \mathrm{CuBr}$ in methanolic acetic acid at $0{ }^{\circ} \mathrm{C}$ to give 13 in $47 \%$ yield (Scheme 3). Compound 13 was previously reported by Russian workers using a different route. ${ }^{7}$

\section{Experimental Section}

General Procedures. Melting points are uncorrected. All NMR spectra were recorded on a Varian VXR-300 spectrometer at $300 \mathrm{MHz}\left({ }^{1} \mathrm{H}\right)$ and $75 \mathrm{MHz}\left({ }^{13} \mathrm{C}\right)$ in $\mathrm{CDCl}_{3}$, unless otherwise specified. Column chromatography was carried out on Merck Kiesel gel 60 (5386) silica gel. THF was used immediately after distilling from a solution containing benzophenone/sodium. Other starting materials, reagents and solvents were used as received from suppliers.

4-Iodobenzaldehyde (2). A solution of $\mathrm{NaBH}_{4}(129 \mathrm{mg}, 3.4 \mathrm{mmol})$ in dry pyridine $(2 \mathrm{~mL})$, dry DMF $(5 \mathrm{~mL})$ and dry THF $(3 \mathrm{~mL})$ was stirred at $0{ }^{\circ} \mathrm{C}$. 4-Iodobenzoyl chloride (1.06 g, $\left.4 \mathrm{mmol}\right)$ in dry THF $(2 \mathrm{~mL})$ was added rapidly to the solution. The mixture was vigorously stirred at $0{ }^{\circ} \mathrm{C}$ 
for $1 \mathrm{~min}$, at which time $\mathrm{H}_{2} \mathrm{O}(0.5 \mathrm{~mL})$ was added. Stirring was continued at $0{ }^{\circ} \mathrm{C}$ for an additional $1 \mathrm{~min}$, and a mixture of hexane: diethyl ether $(50 \mathrm{~mL}, 4: 1 \mathrm{v} / \mathrm{v})$ was quickly added. The globules of pyridine borane which formed at this point were separated by rapid filtration of the reaction mixture through a silica gel bed. Diethyl ether $(25 \mathrm{~mL})$ was added to the filtrate, and the organic phase was washed successively with $15 \%$ aqueous $\mathrm{NaCl}(2 \times 100 \mathrm{~mL}), 2 \mathrm{M}$ aqueous hydrochloric acid: brine (100 mL, 1:1 v/v), $1 \mathrm{M}$ aqueous Noah : brine $(2 \mathrm{x} 100 \mathrm{~mL}, 4: 1 \mathrm{v} / \mathrm{v})$ and saturated brine $(100 \mathrm{~mL})$. The organic layer was then dried over anhydrous $\mathrm{MgSO}_{4}$, and the solvent was removed in vacuo. The crude product was purified by column chromatography (eluent - hexane: ethyl acetate, 1:1). Yield 53\%, mp 75-78 ${ }^{\circ} \mathrm{C}$ (lit. ${ }^{8} \mathrm{mp} 77-78{ }^{\circ} \mathrm{C}$ ); ${ }^{1} \mathrm{H}$ NMR $\delta$ $\left(\right.$ DMSO-d $\left._{6}\right) 7.67$ (d, 2H, $\left.J=8.2 \mathrm{~Hz}\right), 8.02$ (d, 2H, $\left.J=8.2 \mathrm{~Hz}\right), 9.97$ (s, 1H); ${ }^{13} \mathrm{C}$ NMR $\delta$ (DMSOd6) $103.4,130.9,135.3,138.1,192.6$.

1,4-Bis-(4-iodophenyl)-prop-2-enone (3a). 4-Iodoacetophenone ( $0.1 \mathrm{~g}, 0.41 \mathrm{mmol})$ was added under stirring to a solution of $\mathrm{NaOH}(0.1 \mathrm{~g}, 2.50 \mathrm{mmol})$ in $\mathrm{EtOH}(10 \mathrm{~mL})$ at $20{ }^{\circ} \mathrm{C}$ and stirred for $10 \mathrm{~min}$. A solution of 4-iodobenzaldehyde $(0.1 \mathrm{~g}, 0.43 \mathrm{mmol})$ in $\mathrm{EtOH}(5 \mathrm{~mL})$ was added, and the mixture stirred at $20{ }^{\circ} \mathrm{C}$ overnight. The precipitate was collected by filtration and air-dried. Purification by flash chromatography (chloroform) gave 3a, yield 45\%, mp 218-220 ${ }^{\circ} \mathrm{C}$ (lit. ${ }^{9} \mathrm{mp}$ 219.5-220 ${ }^{\circ} \mathrm{C}$ ).

Ethyl dodecylimino ester hydrochloride (5). A mixture of dodecanonitrile 4 (1.0 g, $5.5 \mathrm{mmol})$ and $\mathrm{EtOH}(5 \mathrm{~mL})$ was treated with hydrogen chloride for $1 \mathrm{~h}$ at $0{ }^{\circ} \mathrm{C}$ and then left to stand overnight at $20^{\circ} \mathrm{C}$. The solvent was evaporated, and the crude product was immediately used in the next step without further purification. Yield $76 \%, \mathrm{mp} 82-84{ }^{\circ} \mathrm{C} ;{ }^{1} \mathrm{H}$ NMR $\delta 0.85-0.88(\mathrm{~m}$, $3 \mathrm{H}), 1.25-1.40(\mathrm{~m}, 16 \mathrm{H}), 1.48(\mathrm{t}, 3 \mathrm{H}, J=6.6 \mathrm{~Hz}), 1.70-1.80(\mathrm{~m}, 2 \mathrm{H}, J=2.0 \mathrm{~Hz}), 2.73(\mathrm{t}, 2 \mathrm{H}, J=$ $7.2 \mathrm{~Hz}$ ), 4.62 (q, 2H, $J=6.7 \mathrm{~Hz}), 11.45$ ( br s, $1 \mathrm{H}), 12.36$ (br s, $1 \mathrm{H}) ;{ }^{13} \mathrm{C} \mathrm{NMR} \delta 13.5,14.0$, 22.6, 25.7, 28.7, 28.9, 29.2, 29.4, 29.5, 31.8, 33.0, 70.5, 179.3.

Dodecylamidine hydrochloride (6). A mixture of ethyl dodecylimino ester hydrochloride 5 $(1.0 \mathrm{~g}, 4 \mathrm{mmol})$ in EtOH $(5 \mathrm{~mL})$ was added to a cold $\left(-70{ }^{\circ} \mathrm{C}\right)$ stirred solution of EtOH $(10 \mathrm{~mL})$ saturated with ammonia over a 5 min interval. The mixture was stirred at $0{ }^{\circ} \mathrm{C}$ for $4 \mathrm{~h}$ and left stand at $20{ }^{\circ} \mathrm{C}$ overnight. The precipitate was collected by filtration and washed with hexanes. Yield 79\%, mp 117-119 ${ }^{\circ} \mathrm{C} ;{ }^{1} \mathrm{H}$ NMR $\delta 0.89$ (t, 3H, $\left.J=5.7 \mathrm{~Hz}\right), 1.15-1.45$ (m, $\left.16 \mathrm{H}\right), 1.71-1.73$ $(\mathrm{m}, 2 \mathrm{H}), 2.60(\mathrm{t}, 2 \mathrm{H}, J=7.3 \mathrm{~Hz}), 8.60$ (br s, $2 \mathrm{H}), 8.72$ (br s, $2 \mathrm{H}) ;{ }^{13} \mathrm{C}$ NMR $\delta 14.1,22.7,26.9$, 29.0, 29.3, 29.4, 29.6, 29.7, 31.9, 32.6, 172.0; HRMS calcd for $\mathrm{C}_{12} \mathrm{H}_{27} \mathrm{~N}_{2} \mathrm{Cl} 199.2174(\mathrm{M}+1)$, found 199.2138 .

2-Dodecyl-4,6-bis-(4-iodophenyl)pyrimidine (7a). Sodium hydroxide (0.24 g, 6 mmol) was added to a vigorously stirred solution of dodecane amidine hydrochloride $(1.4 \mathrm{~g}, 6 \mathrm{mmol})$ in DMSO $(20 \mathrm{~mL})$. The mixture was stirred at $20{ }^{\circ} \mathrm{C}$ for $10 \mathrm{~min}$, then diiodochalcone $3 \mathrm{a}(1.0 \mathrm{~g}$, $2.2 \mathrm{mmol})$ and $5 \AA$ molecular sieves $(5 \mathrm{~g})$ were added. The reaction mixture was heated at $80{ }^{\circ} \mathrm{C}$ for $18 \mathrm{~h}$ with an air stream bubbled through. The molecular sieves were removed by filtration of the warm reaction mixture. On cooling, the product precipitated and was collected by filtration. The crude product was purified by column chromatography (hexane : ethyl acetate, 2:1). Yield $72 \%, \mathrm{mp} 113.0-114.5{ }^{\circ} \mathrm{C} ;{ }^{1} \mathrm{H}$ NMR $\delta 0.88$ (t, 3H, $\left.J=6.5 \mathrm{~Hz}\right), 1.15-1.50$ (m, 16H), 1.90-1.94 (m, 
2H), $3.05(\mathrm{t}, 2 \mathrm{H}, J=7.5 \mathrm{~Hz}), 7.75-8.00(\mathrm{~m}, 9 \mathrm{H}) ;{ }^{13} \mathrm{C}$ NMR $\delta$ 14.1, 22.7, 28.5, 29.3, 29.5, 29.5, 29.6, 29.6, 31.9, 39.7, 97.4, 109.1, 128.8, 136.9, 138.0, 163.8, 172.1. Anal. Calcd for $\mathrm{C}_{27} \mathrm{H}_{31} \mathrm{~N}_{2}$ : C, 50.87; H, 4.90, N, 4.39. Found: C, 51.2, H, 5.2, N, 4.33.

2-Undecyl-4,6-bis-(4-chlorophenyl)pyrimidine (7b). Prepared similarly from dichlorochalcone $3 \mathbf{b}(1.2 \mathrm{~g}, 4.4 \mathrm{mmol})$. Yield 63\%, mp $74-75^{\circ} \mathrm{C} .{ }^{1} \mathrm{H}$ NMR $\delta 0.86(\mathrm{t}, 3 \mathrm{H}, J=6.67 \mathrm{~Hz}), 1.25-1.57$ (m, 16H), 1.91-2.0 (m, 2H), 3.05 (t, 2H, $J=7.69 \mathrm{~Hz}), 7.48(\mathrm{~d}, 4 \mathrm{H}, J=8.52 \mathrm{~Hz}), 7.81(\mathrm{~s}, 1 \mathrm{H})$, $8.08(\mathrm{~d}, 4 \mathrm{H}, J=8.52 \mathrm{~Hz}) ;{ }^{13} \mathrm{C}$ NMR $\delta$ 14.1, 22.7, 28.6, 29.4, 29.5, 29.5, 29.6, 31.9, 39.7, 109.3, 128.5, 129.1, 135.9, 136.9, 163.6, 172.1. Anal. Calcd for $\mathrm{C}_{27} \mathrm{H}_{31} \mathrm{~N}_{2} \mathrm{Cl}_{2}: \mathrm{C}, 71.35 ; \mathrm{H}, 6.87 ; \mathrm{N}$, 6.16. Found: C, 71.24, H, 7.47; N, 6.16 .

3,6-Bis-(4-aminophenyl)-1,2-dihydro-1,2,4,5-tetrazine (10). A solution of 4-nitrobenzonitrile $(2.0 \mathrm{~g}, 13.5 \mathrm{mmol})$ and ammonium sulfide in ethanol $(20 \mathrm{~mL})$ was heated on a steam bath for $24 \mathrm{~h}$. The precipitate was removed by filtration, and the solvent concentrated under reduced pressure. The residue was recrystallized from water. Yield $60 \%, \mathrm{mp} 84{ }^{\circ} \mathrm{C}\left(\right.$ lit. $\left.{ }^{5} \mathrm{mp} 85{ }^{\circ} \mathrm{C}\right)$.

Anhydrous hydrazine $(98 \%, 5 \mathrm{~mL})$ was added to 4 -aminobenzonitrile (9) (1.0 g, $8.4 \mathrm{mmol})$. The solution was heated on a steam bath for $18 \mathrm{~h}$. After cooling, the resulting orange-yellow precipitate was filtered, washed with water and immediately used in the next step without purification. Yield 51\%, mp $261{ }^{\circ} \mathrm{C} ;{ }^{1} \mathrm{H}$ NMR $\delta\left(\mathrm{DMSO}_{6}\right) 5.47\left(\mathrm{~s}, 4 \mathrm{H}, \mathrm{NH}_{2}\right), 6.53(\mathrm{~d}, 4 \mathrm{H}, J=$ $8.3 \mathrm{~Hz}$, arom $\mathrm{H}), 7.47(\mathrm{~d}, 4 \mathrm{H}, J=8.3$, arom $\mathrm{H}), 8.51(\mathrm{~s}, 2 \mathrm{H}, \mathrm{NH}) ;{ }^{13} \mathrm{C}$ NMR $\delta\left(\mathrm{DMSO}-d_{6}\right) 113.0$, 117.3, 126.9, 148.6, 150.4. HRMS calcd for $\mathrm{C}_{14} \mathrm{H}_{14} \mathrm{~N}_{6} 267.1371(\mathrm{M}+1)$, found : 267.1371.

3,6-Bis-(4-aminophenyl)-1,2,4,5-tetrazine (11). A solution of 3,6-bis-(4-aminophenyl)-1,2dihydrotetrazine $(0.5 \mathrm{~g}, 1.8 \mathrm{mmol})$ and aqueous hydrogen peroxide $(4 \%, 50 \mathrm{~mL})$ was warmed at $60{ }^{\circ} \mathrm{C}$ for $2 \mathrm{~h}$, to yield a red solid, which was collected by filtration and air-dried. The crude product was purified by column chromatography on silica gel using acetone and hexane $(2: 1)$ as eluent. Yield 18\%, mp 288-289 ${ }^{\circ} \mathrm{C} ;{ }^{1} \mathrm{H}$ NMR $\delta\left(\mathrm{DMSO}_{6}\right) 6.01\left(\mathrm{~s}, 4 \mathrm{H}, \mathrm{NH}_{2}\right), 6.73(\mathrm{~d}, 4 \mathrm{H}, J=$ $8.7 \mathrm{~Hz}$, arom $\mathrm{H}), 8.15$ (d, $4 \mathrm{H}, J=8.7 \mathrm{~Hz}$, arom $\mathrm{H}) ;{ }^{13} \mathrm{C}$ NMR $\delta$ (DMSO-d 6 ) 113.8, 118.3, 128.5, 152.8, 162.3; HRMS calcd for $\mathrm{C}_{14} \mathrm{H}_{12} \mathrm{~N}_{6} 265.1201$ [M+1], found: 265.1207.

3,6-Bis-(4-iodophenyl)-1,2,4,5-tetrazine (12). Sodium nitrite $(0.10 \mathrm{~g}, 4.8 \mathrm{mmol})$ in water $(0.8 \mathrm{~mL})$ was added within $5 \mathrm{~min}$ to a solution of 3,6-bis-(4-aminophenyl)-1,2,4,5-tetrazine $(0.1 \mathrm{~g}, 0.37 \mathrm{mmol})$ in concentrated hydrochloric acid $(1 \mathrm{~mL})$ and ice $(1.0 \mathrm{~g})$ at $0{ }^{\circ} \mathrm{C}$ while stirring. The mixture was stirred at $0{ }^{\circ} \mathrm{C}$ for a further $20 \mathrm{~min}$, then was placed into a jacketed addition funnel at $0{ }^{\circ} \mathrm{C}$, and was added over $20 \mathrm{~min}$ to a stirred solution of potassium iodide $(2.45 \mathrm{~g}, 0.015 \mathrm{~mol})$ in water $(3 \mathrm{~mL})$ at $20{ }^{\circ} \mathrm{C}$. The mixture was left to stand at $20{ }^{\circ} \mathrm{C}$ overnight and then extracted with ether $(2 \times 30 \mathrm{~mL})$. The combined organic extracts were washed successively with $10 \%$ aqueous $\mathrm{NaOH}(30 \mathrm{~mL}), 5 \% \mathrm{NaHCO}_{3}(30 \mathrm{~mL})$, and $\mathrm{H}_{2} \mathrm{O}(50 \mathrm{~mL})$ and dried over anhydrous $\mathrm{MgSO}_{4}$. Concentration in vacuo and flash chromatography of the residue (hexane: ethyl acetate, 2:1) gave 3,6-bis-(4-iodophenyl)-1,2,4,5-tetrazine (5). Yield 30\%, mp $278{ }^{\circ} \mathrm{C} ;{ }^{1} \mathrm{H}$ NMR $\delta\left(\mathrm{DMSO}_{6}\right) 7.84(\mathrm{~d}, 4 \mathrm{H}, J=8.4 \mathrm{~Hz}$, arom H), $7.91(\mathrm{~d}, 4 \mathrm{H}, J=8.1 \mathrm{~Hz}$, arom $\mathrm{H}) ;{ }^{13} \mathrm{C}$ NMR $\delta\left(\mathrm{DMSO}-d_{6}\right) 96.3,105.9,127.9$, 137.7. Anal. Calcd for $\mathrm{C}_{14} \mathrm{H}_{8} \mathrm{~N}_{4}$ : C, 35.59; $\mathrm{H}$, 1.66. Found: C, 35.58; H, 2.06. 
3,6-Bis-(4-bromophenyl)-1,2,4,5-tetrazine (13). Sodium nitrite (3.14 g, $0.045 \mathrm{~mol})$ in water $(9.1 \mathrm{~mL})$ was added with stirring over $10 \mathrm{~min}$ to a cold $\left(0^{\circ} \mathrm{C}\right)$ solution of 3,6-bis-(4aminophenyl)-1,2,4,5-tetrazine $(0.5 \mathrm{~g}, 0.019 \mathrm{~mol})$ in mixture of concentrated sulfuric acid $(4.5 \mathrm{~mL})$, methanol $(2.3 \mathrm{~mL})$, and water $(6.7 \mathrm{~mL})$. The mixture was stirred at $0{ }^{\circ} \mathrm{C}$ for further 30 min and then added over $45 \mathrm{~min}$ to a stirred, warm $\left(60^{\circ} \mathrm{C}\right)$ solution of copper (I) bromide $(0.4 \mathrm{~g}$, $2.8 \mathrm{mmol})$, hydrobromic acid $(48 \%, 1.2 \mathrm{~mL})$, and water $(6.7 \mathrm{~mL})$. At the end of the addition the mixture was refluxed for $1 \mathrm{~h}$, cooled, and filtered. The precipitate was washed with water $(15 \mathrm{~mL})$, and air-dried. Purification by flash chromatography (hexane : ethyl acetate, 2:1) gave 3,6-bis-(4-bromophenyl)-1,2,4,5-tetrazine (6). Yield 47\%, mp $198{ }^{\circ} \mathrm{C}$; (lit. ${ }^{7} \mathrm{mp} \mathrm{288-289}{ }^{\circ} \mathrm{C}$ ); ${ }^{1} \mathrm{H}$ NMR $\delta 7.69(\mathrm{~d}, 4 \mathrm{H}, J=8.3 \mathrm{~Hz}$, arom $\mathrm{H}), 8.00(\mathrm{~d}, 4 \mathrm{H}, J=8.5 \mathrm{~Hz}$, arom $\mathrm{H}) ;{ }^{13} \mathrm{C}$ NMR $\delta 122.7$, 126.6, 128.3, 132.5, 164.0. Anal. Calcd. for $\mathrm{C}_{14} \mathrm{H}_{8} \mathrm{~N}_{4}$ : C, 42.88; H, 2.05. Found: C, 42.88; H, 2.15 .

\section{References and Notes}

1. Wais, A. L.; Shyrina, V. M.; Mamaev, V. P. Izv. Sib. Otd. Akad. Nauk, Ser. Khim. Nauk. 1975, 6, 144 (Russ.); Chem. Abstr. 84:105528r

2. Babler, J. H. Synth. Commun. 1982, 12,839.

3. Dinculescu, A. Rom. Pat. 59061 (Cl. CO7C123/00), 07 Jul 1975; Chem. Abstr. 1978, 88, $169615 \mathrm{~g}$.

4. Pinner, A. Liebigs Ann. Chem. 1897, 297, 221.

5. Guither, W.; Coburn, M. Heterocycles 1979, 12, 745.

6. Clive, D. L.; Angoh, A. G. J. Org. Chem. 1987, 52, 1339.

7. Matyushecheva, G. I.; Mikhailov, V. S.; Yagupol'skii, L. M. Zh. Org. Khim. 1974, 10, 124.

8. Wheeler, O. H. Can. J. Chem. 1958, 36, 667.

9. Neunhoeffer, O.; Rosahl, D. Chem. Ber. 1953, 86, 226. 